\title{
Selection of Repetition Codes for MAC in Vehicular Ad Hoc Networks
}

\author{
Ali Honarvar \\ School of Electrical and \\ Computer Engineering \\ University of Toronto \\ Toronto, Ontario, M5S 2E4, Canada \\ Email: honarvar@comm.toronto.edu
}

\author{
Shahrokh Valaee \\ School of Electrical and \\ Computer Engineering \\ University of Toronto \\ Email:valaee@comm.utoronto.ca
}

\begin{abstract}
In the context of vehicular safety and entertainment applications, we focus on the design of a reliable medium access control scheme. Each vehicle is willing to form a network and regularly communicate with the other vehicles in its vicinity, forming a Vehicular Ad hoc Network (VANET). The information that is being communicated is short lived, and requires a very low probability of failure. In this article we assume that there is no central entity managing the medium access for the cluster of vehicles.

Several code-based repetition-based QoS provisioning MAC schemes have been proposed for this scenario. We present a mathematical description of the frame failure probability, our performance metric, and explain its application in finding optimal codes.
\end{abstract}

\section{Introduction}

Short-range communications (below 500 meters) can be used in vehicular networks using IEEE 802.11 protocols, specifically WAVE or the Dedicated Short Range Communications standard [1] adopted by IEEE and ASTM International (ASTM E 2213-03 [2] ) and currently being promoted by the Intelligent Transportation Society of America and the United States Department of Transportation (also refer to SAE J2354 [3]). Longer range communications have been proposed using infrastructure networks such as IEEE 802.16 (WiMax), GSM or 3G. Long-range communications using these methods are well established, but they usually require extensive infrastructure deployment. In the DSRC standard, $75 \mathrm{MHz}$ bandwidth at $5.9 \mathrm{GHz}$ is allocated to public and private vehicular communication applications [4]. The $75 \mathrm{MHz}$ bandwidth is divided into seven $10 \mathrm{MHz}$ channels. Among the seven designated channels, one channel is the control channel (ch 178) used mainly for broadcast traffic. Our focus is the Medium Access Control protocol (MAC layer) in ad hoc mode for broadcast communication.

The MAC protocol must be able to reliably deliver safetycritical messages. Due to stringent delay requirements of safety traffic, transmission delay of a protocol designed for vehicular communication must be very low. Furthermore, a vehicular MAC must be capable of supporting mobility and effectively coordinating tens of sources of broadcast type traffic.

The ad hoc MAC protocols presented here are topology transparent protocols that use repetition codes as their channel assignment patterns as explained later in $\S$ I-B A few examples of the repetition-based broadcasting schemes for vehicular communications have been proposed [5], [6], and [7].

In this work, we provide a detailed analysis for probability of failure of topology-transparent protocols, and propose optimal code-based schemes. We also study merits and drawbacks of a few code-based repetition-based systems and compare them to codeless approaches.

The rest of this article is organized as follows. We present the motivation and justify the problem in $\S$ I-A In $\S$ I-B problem formulation is presented. We present our performance metric in $\S$ II and show how we separate channel, code and load. Next we introduce the applications in $\S$ II-C1 and $\S$ II-C2 We present proofs for some of the theorems in $\S$ III and provide a conclusion in $\S$ IV

\section{A. Motivation}

There are two main approaches for repetition based MAC for VANETs. One is based on "codes" assigned to vehicles in a cluster. Each code shows the time-slot in a time-frame during which the owner of the code is allowed to broadcast, should he have anything to send. In a codeless scheme, there are rules that govern how a time-slot is randomly picked for broadcasting by a vehicle. In a code-based scheme before a vehicle can start sending its messages, it has to "register" itself by picking up a code. The code should not have many potential collisions with the codes of other vehicles in the vicinity. When a vehicle joins a cluster of vehicles, or two clusters merge together, they have to make sure that the codes in the new cluster are not stepping on each other two often. In other words, a phase of checking and/or registration ensues a merge. A mechanism to free inactive codes should also be in place. A codeless repetition-based scheme does not require any "code-acquisition" part and therefore is easier to form. On the other hand, the performance of such system might be inferior to that of a code-based one.

Previously, randomized (codeless) schemes such as SPR and SFR have been proposed [7] and studied. Also, Positive Orthogonal Codes (formerly Optical Orthogonal Codes, or OOC, since they originally came from the context of Optical CDMA [8]) and constant weight codes have been proposed [5] 
and successfully applied to the problem of MAC in VANETs. The performance of POC has been shown to be superior to that of codeless schemes (SPR, SFR) [9]. To recall how SPR and SFR work, assume that some vehicle are active (have packets to send) in a certain time frame. Each frame has $L$ time-slots. Each active vehicle has to select some of the time-slots of a frame and repeat the same packet in the selected time-slots. In SPR, the selection of each time-slot is done independently and randomly, with probability $p_{0}=\frac{w}{L}$. In SFR, $w$ out of $L$ timeslots are randomly selected. In a code-based scheme, the "l"s in the code that is assigned to a vehicle denotes the time-slots of a frame in which the vehicle is allowed to broadcast.

A problem with previous POC-based codes is that in POCs pairwise correlations of codes are the only major restriction (denoted as $\lambda$ ), which is good enough for a low traffic regime. However, when the probability of several nodes competing for the same time-frame is non-negligible, each one contributes to the packet loss, and it is the correlation of a code with the combined form of several other codes that plays a significant role. We use the common metric of frame failure probability, and study the performance of different schemes under this metric. We will analytically see that only in low traffic regime the performance metric is related to $\lambda$.

In addition, we note that with the same parameters, one can have different codebooks. Although these codebooks are similar in the context of Optical CDMA, they do not have the same performance in the context of repetitive broadcasting MAC protocols. For instance consider the following example, for $n=3$ users, frame-size of $L=7$ time-slots, and $w=3$ active time-slots in a frame for each vehicle. Both codebooks $A_{1}$ and $A_{2}$ are POC codebooks, but their performance is different as shown in figure I-A. The codebooks differ in their last row. Each row in a codebook matrix is a code that can be assigned to a vehicle. Imagine the first two rows are assigned to two vehicles already in a network. If we use the codebook $A_{2}$, the network will have lower performance degradation after the third node joins in. This leaves the actual parameters that control the performance to be studied.

$$
\begin{aligned}
A_{1} & =\left[\begin{array}{lllllll}
1 & 1 & 0 & 0 & 1 & 0 & 0 \\
1 & 0 & 1 & 0 & 0 & 1 & 0 \\
0 & 1 & 1 & 0 & 0 & 0 & 1
\end{array}\right] \\
A_{2} & =\left[\begin{array}{lllllll}
1 & 1 & 0 & 0 & 1 & 0 & 0 \\
1 & 0 & 1 & 0 & 0 & 1 & 0 \\
1 & 0 & 0 & 1 & 0 & 0 & 1
\end{array}\right]
\end{aligned}
$$

There is also another question: Are POC codebooks the optimal choice if one can use any code-based MAC scheme. The answer is, in fact, negative. An example shows that the choice of the optimal codebook depends on the traffic load, and also on channel erasure probability. Furthermore, POC is

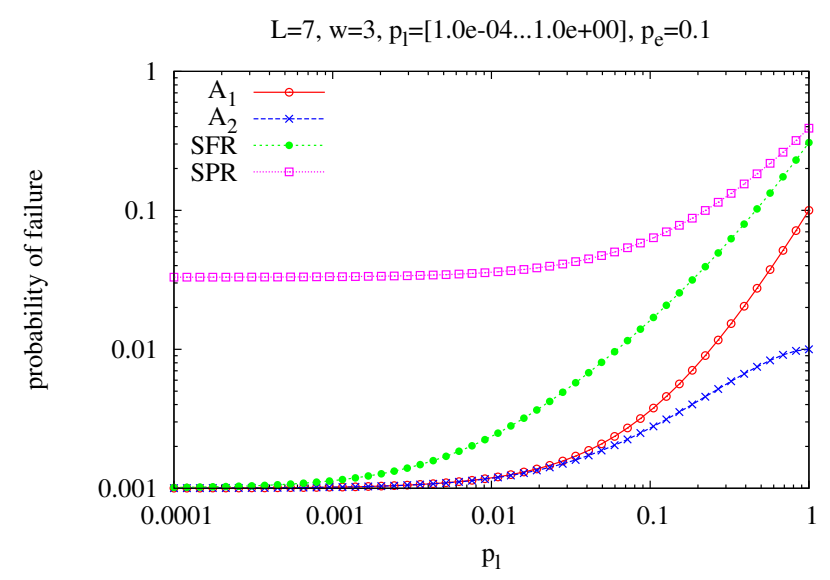

Fig. 1. Performance $\left(\bar{P}_{f}\right.$, as defined in $\S$ II comparison between two different codes.

non-optimal for a wide range of possible values of parameters.

$$
\begin{aligned}
A_{441111} & =\left[\begin{array}{llllll}
1 & 1 & 1 & 0 & 0 & 0 \\
1 & 1 & 0 & 1 & 0 & 0 \\
1 & 1 & 0 & 0 & 1 & 0 \\
1 & 1 & 0 & 0 & 0 & 1
\end{array}\right] \\
A_{222222} & =\left[\begin{array}{llllll}
1 & 1 & 1 & 0 & 0 & 0 \\
1 & 0 & 0 & 1 & 1 & 0 \\
0 & 1 & 0 & 1 & 0 & 1 \\
0 & 0 & 1 & 0 & 1 & 1
\end{array}\right]
\end{aligned}
$$

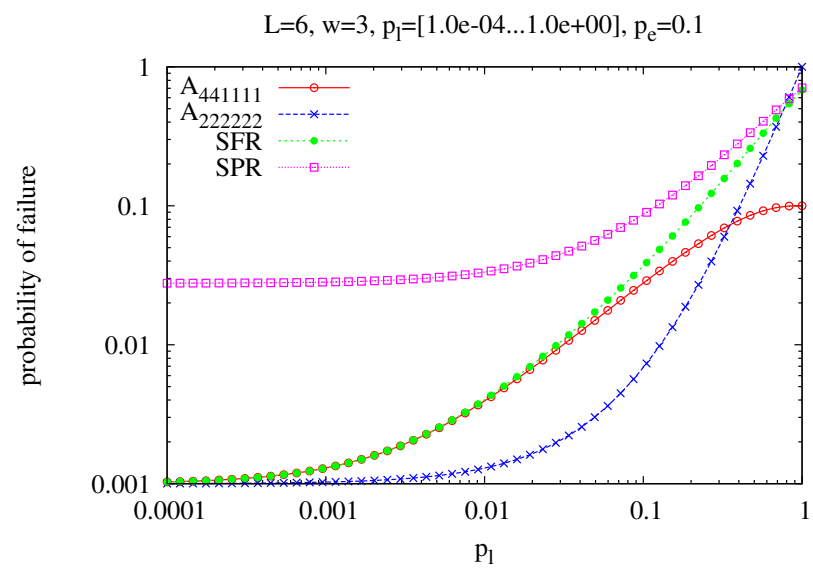

Fig. 2. Performance $\left(\bar{P}_{f}\right)$ comparison between two different codes.

As you can see in figure I-A for low load (e.g., when for load $p_{l}<0.35$ in the upper graph) the code denoted $A_{222222}$ has a better performance. However when the load is high, the code $A_{441111}$ which has the maximum cross correlation $\lambda=2$ performs better than $A_{222222}$ which has $\lambda=1$. It is apparent that for a high load regime having a low $\lambda$ is not helpful; give 
the same resources (mainly $n, L$ ), it is better to sacrifice $\lambda$, and try to free up more "singly booked" time-slots.

There are other applications for repetition based MAC schemes. In networks where nodes generate geo-significant short-lived "updates", or for other reasons acknowledgements cannot be used, repetition based MAC protocols can improve the reliability of the communication. There are several applications where nodes broadcast the changes they have made to the common (world) state. (virtual reality, ubiquitous computing, wireless gaming, field communication, etc.) It is often desirable that the lost packets be piggy backed onto consecutive packets, but the overhead forbids any coordination for retransmission within the same frame.

\section{B. Problem Formulation}

We assume a vehicular ad hoc network (VANET). This type of network has the following characteristics: Power consumption is not a limiting factor. Processing and computation power can be moderate or high. Probability of packet loss should be very low. The network has a highly dynamic topology with known vehicle mobility models Additionally, we require a distributed medium access control.

For certain safety applications, each vehicle has to broadcast its messages to nearby vehicles, regularly. This leaves little room for feedback and acknowledgement-based (ACK) delivery insurance. The main communication scheme is often broadcasting 1 However, these applications usually require a very low packet loss probability. In the absence of ACK information, repetition seems to be a plausible choice.

\section{Probability of Frame Failure}

We define the probability of frame failure $\left(P_{f}\right)$ as follows. A message is sent in a frame being repeated in multiple time-slots in that frame. Consider one of the vehicles. The message is lost if that vehicle does not detect any of the message's time-slots by the end of the frame. In this article, we focus on optimizing probability of frame failure, as the main metric. Note that an POC set is not a set of orthogonal codes. Therefore, it does matter if there is only one contender or multiple contenders when one is trying to broadcast a message. As we will see, computing frame failure probability is more complex than just looking at the maximum cross correlation of two codes $(\lambda)$.

For a given load $\left(p_{l}\right)$, a common channel erasure probability $\left(p_{e}\right)$, and a given repetition scheme, we define the average probability of frame failure $\left(\overline{P_{f}}\right)$ as the probability with which there is a node that does not receive any of the messages of another node in a time-frame.

To explain it further, assume node $a$ is active in a timeframe. It will repeat its message in some time-slots. In some of these time-slots $a$ is the only one broadcasting (call them $a$ 's broadcasting opportunities). $a$ 's other time-slots are lost because other nodes are also broadcasting. $\overline{P_{f}}$ is the probability that there is a node, in the cluster of nodes, that loses all $a$ 's broadcasting opportunities.

\footnotetext{
${ }^{1}$ One can think of piggy-backed ACKs, which might be helpful for certain types of traffic, but it is less useful for safety messages where one vehicle cannot wait until all ACKs are collected.
}

\section{A. Separation of Channel, Load and Code}

We separate the frame failure probability $\left(\overline{P_{f}}\right)$ into three parts.

- Vector $P_{l}$ : depends on the network load (traffic) of the cluster. (binomial distribution of interfering nodes)

- Vector $P_{e}$ : depends on the physical layer channel parameters (e.g., erasure probability, for this article).

- Matrix $S$ : only depends on the repetition algorithm (e.g., the code that is used in a code-based scheme). We call this part the structure matrix of the repetition scheme.

\section{Theorem 1.}

$$
\overline{P_{f}}=P_{e}^{\mathrm{T}} S P_{l}
$$

where,

$$
P_{l}=\left[\begin{array}{c}
\left(\begin{array}{c}
n-1 \\
0
\end{array}\right) p_{l}^{0}\left(1-p_{l}\right)^{n-1} \\
\left(\begin{array}{c}
n-1 \\
1
\end{array}\right) p_{l}^{1}\left(1-p_{l}\right)^{n-2} \\
\vdots \\
\left(\begin{array}{l}
n-1 \\
n-1
\end{array}\right) p_{l}^{n-1}\left(1-p_{l}\right)^{0}
\end{array}\right] \quad P_{e}=\left[\begin{array}{c}
p_{e}^{0} \\
p_{e}^{1} \\
\vdots \\
p_{e}^{L}
\end{array}\right]
$$

- For a code-based scheme, we have:

$$
S_{k, m}=\frac{1}{n\left(\begin{array}{c}
n-1 \\
m
\end{array}\right)} \sum_{a \in A_{0}} \sum_{\substack{A \subset A_{0}-\{a\} \\
|A|=m \\
\|A \cup\{a\}\|-\|A\|=k}} 1 .
$$

- For SPR we have:

$$
S_{k, m}=\left(\begin{array}{l}
L \\
k
\end{array}\right)\left(p_{0}\left(1-p_{0}\right)^{m}\right)^{k}\left(1-p_{0}\left(1-p_{0}\right)^{m}\right)^{L-k}
$$

for $k=0,1, \cdots, L$, and, $m=0,1, \cdots,(n-1)$.

- For SFR we have:

$$
S_{k, m}=\sum_{y=k}^{w}(-1)^{y-k}\left(\begin{array}{c}
w \\
w-y, y-k, k
\end{array}\right)\left(\frac{\left(\begin{array}{c}
L-y \\
w
\end{array}\right)}{\left(\begin{array}{c}
L \\
w
\end{array}\right)}\right)^{m},
$$

for $k=0,1, \cdots, w$, and, $m=0,1, \cdots,(n-1)$.

For the proofs refer to $\S$ III-A (for the code-based part), $\S$ III-C (for SPR), and $\S$ III-D (for SFR). 2

\section{B. Properties of the structure matrix $(S)$}

Each column of $S$ is the conditional probability distribution of the number of broadcasting opportunities (conditioned on the number of active contenders). Other than that, $S$ has the following properties.

Property 1 (Maximum Pair-wise Correlation). Note that maximum pair-wise correlation ( $\lambda$, as defined in some of the previous works) of codes in $A_{0}$ can be seen in S. Specifically, $S_{1, w-1+\lambda}$ is where the last 0 in column 1 (second column) of $S$ appears.

\footnotetext{
${ }^{2}$ Note that for an alternative definition of frame failure, where the message is considered a failure whenever there exists a vehicle who did not detect any of the time-slots of the message, 11 will still hold with a minor change: $P_{e}^{\prime}=(n-1) P_{e}$.
} 
The last columns of $S$ corresponds to very high load. If the load is 1 , only the last matters. In fact, the last column is the distribution of the number of broadcasting opportunities when load is one.

Property 2 (Effective $L$ ). In code-based schemes, the value of $L_{\mathrm{eff}}$, number of occupied time-slots, can be obtained from $S$ :

$$
L_{\text {eff }}=\sum_{m=0}^{n-1} \sum_{k=0}^{w} \mathrm{E}_{A}[\operatorname{Pr}\{K \leq k|| A \mid=m\}]
$$

The proof is omitted in this article. In codeless schemes the equation turns to an inequality, with $L_{\text {eff }}$ being an upper limit for the right hand side in (6).

Property 3 (Constructing $A$ from $S$ ). For simple $S$ where $S$ only consists of " 1 "s and " 0 ", and satisfies other properties, it is possible to find a corresponding $A$.

Property 4 (Other Performance Metrics). $S$ also appears in other performance metrics, for instance, in active throughput, defined as the average number of unique bits of information received by all vehicles in a cluster in a unit of time.

As an example, consider the two codes $A_{1}$ and $A_{2}$ mentioned earlier. Their corresponding structure matrixes are as follows.

$$
\begin{aligned}
& S_{1}=\left[\begin{array}{lll}
0 & 0 & 0 \\
0 & 0 & 0 \\
0 & 1 & 1 \\
1 & 0 & 0
\end{array}\right] \\
& S_{2}=\left[\begin{array}{lll}
0 & 0 & 0 \\
0 & 0 & 1 \\
0 & 1 & 0 \\
1 & 0 & 0
\end{array}\right]
\end{aligned}
$$

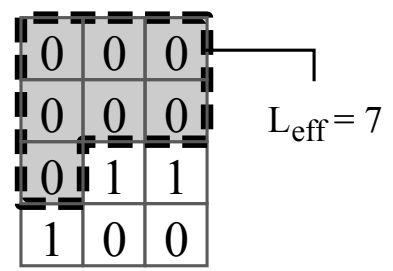

Fig. 3. The area of the upper region is equal to $L_{\text {eff }}$

Computing $S$ directly from (3) has the complexity $\sum_{k=0}^{L} \sum_{m=0}^{n-1} n\left(\begin{array}{c}n-1 \\ m\end{array}\right)=O\left(n L 2^{n}\right)$ which might not be feasible for large $n$. Here we have assumed that checking $\|A \cup\{a\}\|-\|A\|=k$ is a single operation, which is an optimistic assumption. On the other hand, in one of the applications (refer to $\S$ II-C1) we need to compute $S$ many times, which is infeasible for larger values of $n$ given the exponential complexity of (3). To address this issue, we provide the following theorem that reduces the complexity of (3).
Theorem 2. Assume $X_{i}$ is the subset of codes in $A_{0}$ that are inactive in their ith time-slot. Also assume a $(j)$ denotes the $j$ th active time-slot in code $a$. Let $w$ be the maximum weight among all codes in $A_{0}$. For code-based schemes, we have: to

$$
S_{k, m}=\frac{1}{n\left(\begin{array}{c}
n-1 \\
m
\end{array}\right)} \sum_{a \in A_{0}} \sum_{Y \subset \mathcal{Y}(a)}(-1)^{|Y|-k}\left(\begin{array}{c}
|Y| \\
k
\end{array}\right)\left(\begin{array}{c}
\left|R_{a}(Y)\right| \\
m
\end{array}\right),
$$

where

$$
\mathcal{Y}(a)=\left\{X_{a(1)}, X_{a(2)}, \cdots, X_{a(w)}\right\} .
$$

Refer to $\S$ III-B for the proof.

Using (7) the complexity of computing $S$ is $O\left(n^{2} w 2^{w}\right)$, because: There is a summation over all codes ( $n$ code). For each code there are at most $2^{w}$ possible choices of $Y$. For each $Y$, the value $R_{a}(Y)$ is computed with $O(w)$ operations. Then the matrixes $U$ and $V$ are formed and multiplied and added to the summation. This can take $O(n w)$ operations. Note that $w$ is a small integer, for instance $w \leq 5$ is a realistic assumption. Since the complexity of computing is relatively low, it is feasible to have every vehicle compute it when they want to pick another code.

When the load is high, the last column of $S$ is more important, since $P_{l}$ is larger in its last elements. Intuitively this is because when the load is high, most often the number of active contenders is high, therefore slots that are assigned to only a single code (slots with footprint of 1 ) are increasingly the only ones that succeed.

\section{Applications}

1) Incremental Greedy Code Selection: For given $\left(p_{l}, p_{e}, L\right)$, assume every new vehicle joining the cluster picks the code that minimizes $\overline{P_{f}}$. If the new vehicle uses the same $w$ as previous members, it can find the next code with $O\left(\left(\begin{array}{c}L \\ w\end{array}\right) n 2^{w}\right)$ operations.

2) Optimal Code Selection: The incremental code selection can fall into local maximal points in each step which is not necessarily the global maximum. Simulations show that this happens. For a given set of vehicles, one can compute a totally new set of codes optimized for the given number of vehicles, load and channel parameters. To do this we can select a suitable value for $L$. Then we use the properties of the structure matrix, and find the optimal $S$. The properties of $S$ together with the optimization goal translate to the following optimization problem:

$$
\begin{aligned}
& \min \sum a_{i} p_{e}^{x_{i}} \\
& \text { s.t. } \\
& \quad x_{i} \geq x_{i+1} \geq 0 \\
& \sum x_{i}=L .
\end{aligned}
$$

10) can be turned into a convex optimization problem (use $\left.y_{i}=\log x_{i}-\log x_{i-1}\right)$. We can impose the extra restriction for $x_{i}$ 's to be integer, which results in a simple and fair $S$, i.e., one that only consists of " 0 "s and "1"s. It is easy to find the corresponding $A$ from such $S$. 


\section{Proof of Theorems}

\section{A. Theorem 1, Code-Based Schemes}

Proof: Assume $A_{0}$ is the set of all codes in use in a cluster. It is also a matrix with each vehicle using the code in its corresponding row. Each column represents a certain time-slot in the time-frame.

Let weight of $a$, denoted as $\|a\|$, be the number of " 1 "s in row $a$. Let weight of a set (matrix) be defined as the number of columns that have at least one " 1 ", or equivalently, the weight of the logical-or of the codes in that set.

For a time-frame, assume vehicle $a$ is active (has a message to broadcast). Assume subset $A \subset A_{0}-\{a\}$ is also active (with probability $\left.\left(p_{l}\right)^{|A|}\left(1-p_{l}\right)^{\left|A_{0}\right|-1-|A|}\right)$. The weight difference between $A \cup\{a\}$ and $A$ shows how many of $a$ 's time-slots remain interference-free ( $a$ 's broadcast opportunities). The probability of failure in this situation is $p_{e}^{\|A \cup\{a\}\|-\|A\|}$. We have:

$$
\overline{P_{f}}=\frac{1}{n} \sum_{a \in A_{0}} \sum_{A \subset A_{0}-\{a\}} p_{l}^{|A|}\left(1-p_{l}\right)^{\left|A_{0}\right|-1-|A|} p_{e}^{\|A \cup\{a\}\|-\|A\|}
$$

$$
=\frac{1}{n} \sum_{a \in A_{0}} \sum_{m=0}^{n-1} \sum_{\substack{A \subset A_{0}-\{a\} \\|A|=m}} p_{l}^{m}\left(1-p_{l}\right)^{n-1-m} p_{e}^{\|A \cup\{a\}\|-\|A\|}
$$

$$
=\frac{1}{n} \sum_{a \in A_{0}} \sum_{m=0}^{n-1} \sum_{k=0}^{L} \sum_{\substack{A \subset A_{0}-\{a\} \\|A|=m \\\|A \cup\{a\}\|-\|A\|=k}} p_{l}^{m}\left(1-p_{l}\right)^{n-1-m} p_{e}^{k}
$$

$$
=\frac{1}{n} \sum_{m=0}^{n-1} \sum_{k=0}^{L} p_{l}^{m}\left(1-p_{l}\right)^{n-1-m} p_{e}^{k} \sum_{a \in A_{0}} \sum_{\substack{A \subset A_{0}-\{a\} \\|A|=m \\\|A \cup\{a\}\|-\|A\|=k}} 1
$$

$$
=\sum_{m=0}^{n-1} \sum_{k=0}^{L}\left(\begin{array}{c}
n-1 \\
m
\end{array}\right) p_{l}^{m}\left(1-p_{l}\right)^{n-1-m} p_{e}^{k} S_{k, m}
$$

where, in order to set 11 and $\sqrt{14}$ in agreement, we defined $S$ as:

$$
S_{k, m}=\frac{1}{n\left(\begin{array}{c}
n-1 \\
m
\end{array}\right)} \sum_{a \in A_{0}} \sum_{\substack{A \subset A_{0}-\{a\} \\
|A|=m \\
\|A \cup\{a\}\|-\|A\|=k}} 1
$$

In other words, $n\left(\begin{array}{c}n-1 \\ m\end{array}\right) S_{k, m}$ is the number of ways you can pick a member $a$ and $m$ other members (set $A$ ) from set $A_{0}$ such that the weight difference between $A \cup\{a\}$ and $A$ is equal to $k$.

\section{B. Theorem 2, Code-Based Schemes}

Assume $X_{i}$ is the subset of codes in $A_{0}$ that are inactive in their $i$ th time-slot. Also assume $a(j)$ denotes the $j$ th active time-slot in code $a$. Let $w$ be the maximum weight among all codes in $A_{0}$. Let $\mathcal{Y}(a)=\left\{X_{a(1)}, X_{a(2)}, \cdots, X_{a(w)}\right\}$. Saying $A \subset X_{a(i)}$ is equivalent to saying that $a$ 's $i$ th active time-slot is a broadcasting opportunity. So $\|A \cup\{a\}\|-\|A\|=k$ means that $A$ is a subset of exactly $k$ members of $\mathcal{Y}(a)$.

For each $a$ we are interested in the number of $m$-element sets $A \subset A_{0}-\{a\}$ such that $A$ is the subset of exactly $k$ members of $\mathcal{Y}(a)$. Let $\psi_{k, m}$ be the number of such sets.

Assume that

$$
R_{a}(Y) \triangleq \begin{cases}\bigcap_{X \in Y} X & Y \neq \varnothing \\ A_{0}-\{a\} & Y=\varnothing\end{cases}
$$

Note that $\bigcap_{X \in Y} X$ is the set of codes using $Y$ that will leave at least $k$ free time-slots for $a$. So, $\left(\cap_{X \in Y} X \mid\right)$ is the number of ways you can pick sets of $m$-elements from the above set. When $Y$ is empty it should mean all the codes other than $a$, hence the definition $R_{a}(Y)=A_{0}-\{a\}$ in this case.

\section{Lemma 1.}

$$
\begin{aligned}
& \left|\left\{A: A \subset A_{0}-\{a\},|A|=m,|\{X: X \in \mathcal{Y}(a), A \subset X\}|=k\right\}\right| \\
& =\sum_{Y \subset \mathcal{Y}(a)}(-1)^{|Y|-k}\left(\begin{array}{c}
|Y| \\
k
\end{array}\right)\left(\begin{array}{c}
R_{a}(Y) \\
m
\end{array}\right)
\end{aligned}
$$

Proof: For a set $R$, define $\left(\begin{array}{c}R \\ m\end{array}\right)$ as the set of $m$-elements subsets of set $R$. Note that $\left(\begin{array}{c}|R| \\ m\end{array}\right)=\left|\left(\begin{array}{c}R \\ m\end{array}\right)\right|$. Assume

$$
\Psi_{k, m}=\sum_{Y \subset \mathcal{Y}(a)}(-1)^{|Y|-k}\left(\begin{array}{c}
|Y| \\
k
\end{array}\right)\left(\begin{array}{c}
R_{a}(Y) \\
m
\end{array}\right)
$$

which is a linear combination of sets. We will show that the coefficient of each set is either zero or one. For an $m$-element set $A$, pick the set $Y_{a, A}=\{X: X \in \mathcal{Y}(a), A \subset X\}$, and assume $\left|Y_{a, A}\right|=k_{A}$. Now look at the coefficients of $A$ in $\Psi_{k, m}$. Note that $A$ only appears in the sets $\left(\begin{array}{c}R_{a}(Y) \\ m\end{array}\right)$ when $Y \subset Y_{a, A}$. There are three cases:

1) $k>k_{A}$. In this case $A$ may only appear when $|Y|<$ $\left|Y_{a, A}\right|=k_{A}<k$. But such $Y$ only produces zero coefficients because $|Y|<k$ results in $\left(\begin{array}{c}|Y| \\ k\end{array}\right)=0$.

2) $k=k_{A}$. In this case, only $Y=Y_{a, A}$ may produce a non-zero coefficient. The coefficient is, in fact, $(-1)^{|Y|-k}\left(\begin{array}{c}|Y| \\ k\end{array}\right)=1$.

3) $k<k_{A}$. In this case the only $Y$ 's that may produce 
non-zero coefficients are the subsets of $Y_{a, A}$. We have:

$$
\begin{aligned}
\operatorname{coeff}_{A} & =\sum_{Y \subset Y_{a}, A}(-1)^{|Y|-k}\left(\begin{array}{c}
|Y| \\
k
\end{array}\right) \\
& =\sum_{y=k}^{k_{A}} \sum_{\substack{Y \subset Y_{a}, A \\
|Y|=y}}(-1)^{y-k}\left(\begin{array}{l}
y \\
k
\end{array}\right) \\
& =\sum_{y=k}^{k_{A}}(-1)^{y-k}\left(\begin{array}{c}
y \\
k
\end{array}\right)\left(\begin{array}{c}
k_{A} \\
y
\end{array}\right)=0 .
\end{aligned}
$$

Using (17) we can compute $S_{k, m}$ for $k=0,1, \cdots, w$ and $m=0,1, \cdots,(n-1)$.

$$
S_{k, m}=\frac{1}{n\left(\begin{array}{c}
n-1 \\
m
\end{array}\right)} \sum_{a \in A_{0}} \sum_{Y \subset \mathcal{Y}(a)}(-1)^{|Y|-k}\left(\begin{array}{c}
|Y| \\
k
\end{array}\right)\left(\begin{array}{c}
\left|R_{a}(Y)\right| \\
m
\end{array}\right)
$$

The following reformatting of 22) is possibly more suitable for an implementation.

$$
S=\frac{1}{n} \sum_{a \in A_{0}} \sum_{Y \subset \mathcal{Y}(a)} U(|Y|) V^{\mathrm{T}}\left(\left|R_{a}(Y)\right|\right)
$$

where

$$
U(x)=\left[\begin{array}{c}
(-1)^{x}\left(\begin{array}{l}
x \\
0
\end{array}\right) \\
(-1)^{x-1}\left(\begin{array}{l}
x \\
1
\end{array}\right) \\
\vdots \\
(-1)^{x-w}\left(\begin{array}{l}
x \\
w
\end{array}\right)
\end{array}\right] \quad V(x)=\left[\begin{array}{c}
\left(\begin{array}{c}
x \\
0
\end{array}\right) \\
\left(\begin{array}{c}
n-1 \\
0 \\
x \\
1
\end{array}\right) \\
\frac{\left(\begin{array}{c}
n-1 \\
1
\end{array}\right)}{\vdots} \\
\left(\begin{array}{c}
x \\
n-1
\end{array}\right) \\
\left(\begin{array}{l}
n-1 \\
n-1
\end{array}\right)
\end{array}\right]
$$

\section{C. $S P R$}

Proof: For SPR, assume $p_{0}=\frac{w}{L}$. For each active set $A, a$ gets $k$ opportunities, each with probability $p_{0}\left(1-p_{0}\right)^{m}$, and the rest (that is, $(L-k)$ slots) should be non-opportunities.

\section{SFR}

Proof: For SFR, consider the event that the user $a$ is the only broadcaster in time-slots $i_{1}, i_{2}, \ldots, i_{y}$, regardless of what happens in $a$ 's other time-slots ( $a$ 's other time-slots might or might not succeed). The probability of this event is $\left(\begin{array}{c}L-y \\ w\end{array}\right)^{m} /\left(\begin{array}{c}L \\ w\end{array}\right)^{m}$, and it only depends on $y$ and $m$, not on which specific $y$-subset of $w$ time-slots is being considered. Now using the inclusion and exclusion principle, we obtain (5).

\section{Conclusions}

We defined a suitable failure probability metric and presented the problem of optimal code selection in repetitionbased broadcasting MAC for VANETs. We derived closed form equations that separate the channel parameter (in the form of erasure probability), network traffic (in the form of activity probability, or load), and the code (in code-based schemes) or the scheme itself (in two codeless schemes: SPR and SFR). We identified a "structure matrix" for each scheme.
This matrix appears in the performance equations of all the schemes that we studied. It seems looking into the structure matrix is a useful tool for the analysis of repetition based MAC schemes. We have identified properties of the structure matrix to the point that it is possible to find optimal codes for a given scenario. We have also introduce applications of the theorems in the form of two code selection methods. A formula that leads to fast and feasible calculation of the structure matrix for a given set of codes has been presented.

\section{References}

[1] S. Biswas, R. Tatchikou, and F. Dion, "Vehicle-to-vehicle wireless communication protocols for enhancing highway traffic safety," Communications Magazine, IEEE, vol. 44, no. 1, pp. 74-82, Jan. 2006.

[2] A. E2213-03, "Standard specification for telecommunication and information exchange between roadside and vehicle systems $-5 \mathrm{ghz}$ band dedicated short range communications (dsrc) medium access control (mac) and physical layer (phy) specificifications," ASTM E2213-03, July 2003.

[3] A. T. I. Systems, "Message set for advanced traveler information system (atis)," SAE J2354, Nov 1999, version 2: Published Feb 2004.

[4] J. Zhu and S. Roy, "Mac for dedicated short range communications in intelligent transport system," Communications Magazine, IEEE, vol. 41, no. 12 , pp. 60-67, Dec. 2003.

[5] F. Farnoud, B. Hassanabadi, and S. Valaee, "Message broadcast using optical orthogonal codes in vehicular communication systems," in WINITS '07: The First International Workshop on Wireless Networking for Intelligent Transportation Systems. New York, NY, USA: ACM, 2007, pp. 1-6.

[6] F. Farnoud and S. Valaee, "Repetition-based broadcast in vehicular ad hoc networks in rician channel with capture," in INFOCOM Workshops 2008, IEEE, April 2008, pp. 1-6.

[7] Q. Xu, T. Mak, J. Ko, and R. Sengupta, "Vehicle-to-vehicle safety messaging in dsrc," in VANET '04: Proceedings of the 1st ACM international workshop on Vehicular ad hoc networks. New York, NY, USA: ACM, 2004, pp. 19-28.

[8] F. Chung, J. Salehi, and V. Wei, "Optical orthogonal codes: design, analysis and applications," Information Theory, IEEE Transactions on, vol. 35, no. 3, pp. 595-604, May 1989.

[9] F. Farnoud and S. Valaee, "Reliable broadcast of safety messages in vehicular ad hoc networks," in INFOCOM 2009. The 28th Conference on Computer Communications. IEEE, April 2009, pp. 226-234. 